\title{
Relic streams of photons and neutrinos
}

\author{
V. P. Efrosinin \\ Institute for Nuclear Research, RAS, Moscow, Russia
}

Email address:

vladimir.efrosinin@mail.ru

\section{To cite this article:}

V. P. Efrosinin. Relic Streams of Photons and Neutrinos. American Journal of Modern Physics. Vol. 2, No. 5, 2013, pp. $248-250$. doi: $10.11648 /$ j.ajmp.20130205.12

\begin{abstract}
The possible reasons of features of the nature of neutrino are discussed. The estimation of modern density of the number of neutrinos which differs from traditional calculation methods in thermodynamics is proposed. The obtained results in the order of size will be co-ordinated with calculation in thermodynamics. The explanation of the nature of small neutrino mass is offered.
\end{abstract}

Keywords: Cosmology, Parameters of Neutrino, Relic Streams

\section{Introduction}

In the previous paper [2] neutrino masses were calculated using experimental data on neutrino oscillation [1] Such problem has been solved by means of the left-right gauge group $S U_{2}^{L} \times S U_{2}^{R} \times U_{1}$ following the approach proposed in [3] with some changes.

Parameters of neutrino oscillation including experiments with solar and atmospheric neutrinos, in reactor experiments and accelerator experiments are presented in [1] in form:

$$
\begin{aligned}
& \Delta m_{12}^{2}=7.6 \quad(7.3-8.1) \quad\left[10^{-5} \mathrm{eV}^{2}\right], \\
& \Delta m_{23}^{2}=2.4 \quad(2.1-2.7) \quad\left[10^{-3} \mathrm{eV}^{2}\right], \\
& \sin ^{2} \theta_{12}=0.32 \quad(0.28-0.37), \\
& \sin ^{2} \theta_{23}=0.50 \quad(0.38-0.63), \\
& \sin ^{2} \theta_{13}=0.007 \quad(\leq 0.033) .
\end{aligned}
$$

Using experimental data (1) in [2] following results for masses of neutrino have been received:

$$
\begin{aligned}
& m_{1}=0.0069 \div 0.0073 \mathrm{eV}, \\
& m_{2}=0.0110 \div 0.0116 \mathrm{eV}, \\
& m_{3}=0.0471 \div 0.0532 \mathrm{eV}
\end{aligned}
$$

\section{Main Results}

Phenomenon of small masses of neutrinos appears rather essential. There comes a threshold of stability of discrete character of masses of neutrinos and enough free transition from one generation to another. For comparison we result masses of partners of neutrino on the weak charged currents at which birth oscillations is absent:

$$
\begin{aligned}
& m_{e}=0.511 \mathrm{MeV}, \\
& m_{\mu}=105.7 \mathrm{MeV}, \\
& m_{\tau}=1777 \mathrm{MeV} .
\end{aligned}
$$

There is some affinity of neutrino with photons, including the cosmology plane. The photon is some twirled vhirlwind of energy with spin $S=1$, with zero mass. Neutrino has spin $S=1 / 2$ and very small mass (2). Photon spin keeps this quantum gyroscope so the photon has no mass of rest. Neutrino spin it appears insufficient for such existence. Here it is possible to notice that measurement of electron mass (3) is spent to within $0.1 \mathrm{eV}$. It is possible to consider it and accuracy to what there is an electron birth in the nature. All in the nature approximately, both masses of elementary particles, and symmetry. Relic streams of neutrino in a sense are satellite in relation to relic radiation of photons. Further we will state the approached estimation of modern density of number of neutrinos and antineutrinos of one generation in an ultra relativistic case.

Relic electromagnetic radiation has been opened in 1965 by Penzias and Wilson. This phenomenon in 1946 has been predicted by G. Gamow. The average temperature of relic photons makes [4]

$$
T_{0}=2.725 \pm 0.001 \mathrm{~K} .
$$

The form of a power spectrum coincides with split-hair 
accuracy with the form of Planck's radiation [5,6]. The greatest angular variation of temperature $\delta T / T \sim 10^{-3}$ has bipolar character and speaks movement of the Earth. Relic radiation is isotropic. And relic radiation penetrates all Universe.

The number density of relic photons is equal in the modern universe [5]

$$
n_{\gamma, 0}=2 \frac{\varsigma(3)}{\pi^{2}} T_{0}^{3}=410 \frac{1}{\mathrm{~cm}^{3}} .
$$

Here numerical value of $S$-function is equal

$$
\varsigma(3) \approx 1.2
$$

In the barion stage of evolution of the universe at the temperature $T \geq m_{p}$ there could be a reaction of annihilations of nucleons and antinucleons in rest:

$$
p+\bar{p} \rightarrow \pi^{+}+\pi^{-}+\pi^{0}
$$

(We will give later a substantiation of this reaction). With the subsequent decays:

$$
\begin{aligned}
& \pi^{+} \rightarrow \mu^{+}+v_{\mu}, \\
& \pi^{-} \rightarrow \mu^{-}+\bar{v}_{\mu}, \\
& \pi^{0} \rightarrow \gamma+\gamma .
\end{aligned}
$$

Further is

$$
\begin{aligned}
& \mu^{-} \rightarrow e^{-}+\bar{v}_{e}+v_{\mu}, \\
& \mu^{+} \rightarrow e^{+}+v_{e}+\bar{v}_{\mu} .
\end{aligned}
$$

Annihilation in rest leads to spatial symmetry of born $\gamma$ quanta.

The number of electrons corresponded to number of protons from charge preservation. Annihilation of electrons and positrons occurs in reaction:

$$
e^{-}+e^{+} \rightarrow \gamma+\gamma
$$

According to [7] researches of multiplicity of annihilations of $p \bar{p}$ at small energy are absent. At the same time multiplicity of annihilations sharply increases with $\sqrt{s}$ increases. Multiplicity of annihilations of $e^{+} e^{-}$at

$\sqrt{s} \sim 2 \mathrm{GeV}$ is equal $\sim 3$. We believe as multiplicity of $p \bar{p}$ in rest will be not less than $\sim 3$.

So at a birth of four $\gamma$-quanta three pairs neutrino-antineutrino are born also and burn down steams baryon-antibaryon and electron-positron. Neutrino oscillations level streams of each generation of neutrino.

According to (5) we have modern density of number of neutrino and antineutrino of one type:

$$
n_{v, 0} \cong \frac{1}{4} n_{\gamma, 0}=\frac{1}{4} \times 410 \frac{1}{\mathrm{~cm}^{3}}=102.5 \frac{1}{\mathrm{~cm}^{3}}
$$

Calculation of $n_{v 0}$ in thermodynamics gives [5]

$$
n_{v, 0} \cong 112 \mathrm{~cm}^{-3} \text {. }
$$

Direct detecting of relic neutrinos is insoluble problem at present. So to check up parities (11) and (12) it is not obviously.

Behavior of ultrarelativistic photons and neutrinos is similar. That justifies our approach to definition of modern neutrino density. Especially it proves to be true after recombination.

Results of our research allow us to draw some conclusions.

There is a question why there is a mixing of neutrino generations. First of all it is necessary to understand with what accuracy on masses are born in the nature elementary particles. If masses of neutrino generations differ among themselves in the order of size of this accuracy of their emission generation mixing can occur.

The matrix of neutrino mixing is accepted by analogy to quark mixing taking into account an unitarity principle. Use of in parallel other variant of a mixing matrix will appear useful to calculation of errors of received mixing parameters.

It is necessary to tell that results of present researches of elementary particles bear themselves strong dependence on traditional technology. Not clearly that mainly we define, properties of the mixing matrix or the neutrino nature. Direct measurements of neutrino mass will be hardly probable sensitive at level (2).

It is necessary to draw conclusions on situation with studying of $e^{+} e^{-}$and $p \bar{p}$ annihilations, in particular, multiplicity. First of all we will notice that it would be interesting to compare these reactions at identical $\sqrt{s}$. It is interesting to find difference of rest energy and movement energy in annihilation reactions. Not clearly that mainly we define, properties of the mixing matrix or the neutrino nature.

It is necessary to find a way of research of $p \bar{p}$ annihilations at small $\sqrt{s}$ (multiplicity).

In the given calculation it was not required reactions going unusually and there were no infringements of quantum numbers. In particular there was no influence of the raised density of particles in baryon stages. The sequence of reactions and their relative sections did not change in comparison with modern properties. It testifies in favour of structure of elementary particles to a similar point.

Earlier in [8] we considered the leptonic vertex of electroweak theory:

$$
L^{C C}=-\frac{g}{2 \sqrt{2}} j_{\alpha}^{C C} W^{\alpha}+\text { h.c. }
$$


Where $g$ is the $\mathrm{SU}(2)$ gauge coupling constant and $\mathrm{CC}$ current is

$$
j_{\alpha}^{C C}=2 \sum_{l=e, \mu, \tau} \bar{v} \gamma_{\alpha} l_{L}
$$

Thus the reaction was studied

$$
\begin{gathered}
v_{e}+n \rightarrow e^{-*}+p, \\
e^{-*} \rightarrow e^{-}+\gamma,
\end{gathered}
$$

that is the reaction

$$
v_{e}+n \rightarrow e^{-}+p+\gamma
$$

In [8] the parameters of reaction (15) with an electron off-mass-shall: mass of an exited electron, angular distribution, cross section and so on were studied. It should be noted that the process (15) and reaction

$$
v_{e}+n \rightarrow e^{-}+p
$$

Where $e^{-}$is on the mass shell, are not coherent.

In reaction (18) the leptonic vertex of the Lagrangian (13), (14) is on a mass surface. And in reaction (15) this vertex is half outside of a mass surface. There is a production of an exited (virtual) electron. The experiment demonstrates that thus the leptonic vertex behaves similarly to as well as in reaction (18). Anyway it is not accepted in it to enter any form factors. It is difficult to present that the vertex (13), (14) acts as a certain semiconductor. When in one direction the production of the off-shell electron is possible. And in the order direction there is no production of the neutrino in an exited state. Therefore it is impossible to eliminate and capability of production a virtual neutrino in reverse reaction

$$
e^{-}+p \rightarrow v_{e}^{*}+n
$$

where $v_{e}^{*}$ is neutrino off-mass-shell. And the parameters of this reaction can be like to parameters of reaction (15), including mass an exited neutrino $v_{e}^{*}$, as against $v_{e}$, is outside of a mass surface, and therefore has other nature.

Thus there can be a rather large reduction of cross-section the production exited neutrino, than as contrasted to by reaction (17). As the exited electron rather fast descends from off-mass-shell at production of a photon. And exited neutrino long live as roaming propagator.

\section{References}

[1] T. Schwetz, "Neutrino oscillations: present status and outlook," hep-ph/0710.5027, 2007, pp. 1-6.

[2] V.P. Efrosinin, "What are the neutrino masses. Dark matter", vol. 73. Yadernaya Fizika, 2010, pp.975-977.

[3] H. Fritzsch, "Weak interaction mixing in the six-quark theory," Physical Letters B, vol. 73, issue 3, 1978, pp. 317-322.

[4] J.C. Mather et al., "Calibrator design for the COBE far infrared absolute spectrophotometer (FIRAS)," The Austophysical Journal, vol. 512, 1999, pp. 511-520.

[5] D.S. Gorbunov, V.A. Rubakov, Introduction in the early Universe: The theory of hot Big explosion. World Scientific Publishing Co., 2008.

[6] D.S. Gorbunov, V.A. Rubakov, Introduction in the early Universe: Cosmological perturbations. The inflation theory. World Scientific Publishing Co., 2010.

[7] A.B. Balantekin et al., (Particle Data Group) Journal of Physics, vol. G37, 075021, 2010.

[8] V.P. Efrosinin, hep-th/0904.0542, 2009. 